Because no other sources were found in the scan of the Vela constellation, the coincidence of the now source with the source Vel $X R-1$ is almost a certainty. The flight also puts an upper limit on the X-ray emission from other stars in the region, including the fast pulsar. The maximum flux from these objects in the range 4 to $11 \mathrm{keV}$ is roughly $10^{-9} \mathrm{erg}^{\mathrm{cm}^{-2}} \mathrm{~s}^{-1}$.

Further hints about the origins of X-ray stars have come from an investigation by Gatewood and Sofia of the characteristics of Sco X-1 (Astrophys. J. Lett., 154, L69; 1968), the first non-solar X-ray source to be discovered. Together with Eichhorn, these two authors have already established from an analysis of proper motions that sco $X-1$ (which is a blue star-like object) and two nearby stars are probably part of the Scorpio-Centaurus association of stars. This result is confirmed in their latest paper in which they say that the stars of the association, including the two stars near Seo $X$-1, fit the main sequence-the principal series of stars on the Hertzsprung-Russell plot of spectral type-as a function of absolute magnitude. Seo $X-1$ is an exception, however, obstinately remaining well below the main sequence, in the region occupied by superdense stars. Gatewood and Sofia say this invalidates most models published so far, which require either a main sequence star or a giant, and that Sco $X-1$ must be either a white dwarf or a neutron star. Working back from the proper motions, the ScorpioCentaurus stars seem to be twenty million years old and to have a common starting point in space. Gatewood and Sofia say that for Sco $X-1$ to have this age, mass considerations suggest that it at one time exploded, and that what is seen now is a supernova remnant and possibly a neutron star.

\section{OBSERVATIONAL ASTRONOMY}

\section{Space Money for Ground Telescope}

For a short time at least, the University of Texas will enjoy the third largest optical telescope in the worldthe 107 inch reflector which began operating at McDonald Observatory at the end of last year. Still at work a few hundred feet away is the 30 year old 82 inch reflector which was the second largest telescope in the world until the Hale Telescope at Palomar Mountain was finished in 1947. The observatory is in western Texas, 170 miles from the bright lights of El Paso, and its telescopes are among the largest to make regular observations of the planets. Between 1965 and 1967, for example, 23 per cent of observing time on the 82 inch telescope was devoted to the Moon and planets, compared with an allocation of three per cent at Palomar Mountain.

The new telescope has been built with a grant of $\$ 6$ million from NASA, the National Science Foundation and the University of Texas. Its commissioning just now fits in well with the wish of the planetary astronomy panel of the National Academy of Sciences (Nature, 220, 110; 1968) to see more ground-based telescopes, but the 107 inch telescope is just one step in the right direction. It is also a welcome sign that not all of NASA's money disappears into thin air. (NASA is also contributing to the 88 inch reflector being completed at Mauna Kea, an extinct volcano on Hawaii.) There is solid evidence that expenditure like this is a necessary complement to rocketry-groundbased radio astronomers eventually resolved the conflict between the results of the American and Russian Venus probes, for example (Nature, 220, 420; 1968).

MeDonald's 107 inch telescope has a primary of fused silica, one of the new mirror materials being used in the coming generation of big telescopes and having a coefficient of thermal expansion one sixth that of the 'Pyrex' materials formerly in use. An English mounting was chosen for its advantages with a telescope which is to be operated frequently at the Coudé focus. (In the Coudé system, the beam eventually passes along the polar axis of the mounting, which always remains in a fixed position. This means that the Coudé focus also has a fixed position, and bulky items of equipment can be installed there.) As is becoming the fashion, a prime focus corrector has been designed for the 107 inch telescope by Dr C. G. Wynne of the applied optics group at Imperial College, London. Lack of funds has, however, prevented the corrector from being built.

One problem facing the designers of a telescope for planetary astronomy, which tends to a greater extent than conventional astronomy to be done at a range of wavelengths, is that of choosing the best mirror coatings. The plan was to incorporate two-sided flip-flop secondary mirrors in the design, but because of the expense it has only been possible to make one of the Coudé's mirrors two-sided. One side has an aluminium surface, which does well from $3000 \AA$ to $4500 \AA$ but is poor in the infrared; the other side is silver, good from $4500 \AA$ to the far infrared but poor in the ultraviolet. The primary has to have an aluminium surface.

\section{SOLID STATE \\ Unspectacular Progress}

NEARLY four hundred physicists gathered in Manchester between January 7 and 9 for the Physical Society's sixth annual conference on solid state physics. Most of the participants were from British universities and industry, but a small continental contingent was also present. The conference was designed to cover the full scope of solid state physics and to interest people working in the theory of electrons in solids as well as applied physicists concerned with semiconductor and other solid state devices. There were six separate sessions, each on different branches of the subject.

The general tone of the papers reflected the steady progress that is being made in the subject. One recurring theme was the increasing dependence of solid state physics research-and indeed physics research as a whole - on the development and production of semiconductor devices. There were several lectures on amorphous switching devices, and Sir Nevill Mott described at some length the two basic types--one switching from a high resistanec to a low resistance state by the change of potential across and the other switching on the application of a current pulse without requiring a current to hold it in the low resistance state. This seems to be a new technique which has caught the general interest.

The notion of using synchrotron radiation to obtain a continuous band source encompassing wavelengths from the visible region to the soft $\mathrm{X}$-ray region was put forward and its potential for studying excitation levels in crystals was discussed. New techniques for the application of alternating electric fields or heat 